\section{Berechnung des Positionswinkels eines}

Es seien $\alpha_{1}, \alpha_{2}, \delta_{1}, \delta_{2}$ die als gegeben vorausgesetzten Rektaszensionen und Deklinationen der Sterne $S_{1}$ und $S_{2}$. Mit Hilfe dieser Größen lassen sich die Distanz $S_{1} S_{2}=\Delta$ und die Werte des Positionswinkels ( $p_{1}$ bezw. $p_{2}$ ) des Bogens $S_{1} S_{2}$, bezogen auf $S_{1}$ und $S_{2}$, berechnen. Wenn nun $p_{m}$ den Positionswinkel von $S_{1} S_{2}$, bezogen auf den Mittelpunkt $\left(S_{m}\right)$ dieses Bogens, und $\delta_{m}$ die Deklination von $S_{m}$ bedeutet, so lautet die zur Berechnung von $p_{m}$ allgemein angewandte Formel :

$$
p_{m}=1 / 2\left(p_{1}+p_{2}\right)-1 / 16\left(\mathrm{I}+2 \operatorname{tg}^{2} \delta_{m}\right) \Delta^{2} \sin \mathrm{I}^{\prime \prime} \sin 2 p_{m}
$$

Bei großen Distanzen und polnahen Sternen ist aber diese Formel nicht nur unbequem (weil sie nämlich die Berechnung von $\delta_{m}$ verlangt, und $p_{m}$ als nahe bekannt voraussetzt), sondern sie kann auch zu ziemlich fehlerhaften Werten von $p_{m}$ führen. Die folgende einfache Formel gibt aber $p_{m}$ ganz strenge.

Bezeichnet man den Nordpol des Äquators mit $P$, so geben die Dreiecke $P S_{1} S_{m}$ und $P S_{m} S_{2}$ :

$$
\begin{aligned}
& \sin 1 / 2 \Delta \operatorname{tg} \delta_{m}=\quad \operatorname{ctg} p_{1} \sin p_{m}-\cos p_{m} \cos 1 / 2 \Delta \\
& \sin 1 / 2 \Delta \operatorname{tg} \delta_{m}=-\operatorname{ctg} p_{2} \sin p_{m}+\cos p_{m} \cos 1 / 2 \Delta .
\end{aligned}
$$

Bogens, bezogen auf den Mittelpunkt.

Durch Subtraktion dieser Gleichungen erhält man die gesuchte Formel:

$$
\operatorname{ctg} p_{m}=1 / 2\left(\operatorname{ctg} p_{1}+\operatorname{ctg} p_{2}\right) \cdot \sec 1 / 2 \Delta .
$$

Beispielsweise hat man für die Sterne des zur Bestimmung des Skalenwertes eines Heliometers benutzten PoIbogens

Mittl. Äquinox und Epoche r9o0.

$$
\begin{array}{ll}
\alpha_{1}=165^{\circ} 37^{\prime} 30^{\prime \prime} \circ & \delta_{1}=+86^{\circ} \text { 1 } 0^{\prime} 5^{\prime \prime \prime 。} \\
\alpha_{2}=\text { I } 945542.0 & \delta_{2}=+862524.2 .
\end{array}
$$

Hieraus folgt:

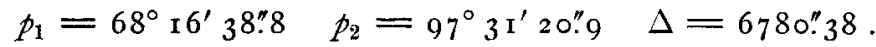

Aus (2) ergibt sich dann der strenge Wert $p_{m}=82^{\circ} 24^{\prime} 47^{\prime \prime} .5$.

\begin{tabular}{|c|c|c|c|c|c|c|c|c|c|c|c|c|c|c|c|c|}
\hline 1912 & \multirow{2}{*}{\multicolumn{2}{|c|}{$\begin{array}{l}\text { M.7.Gr. } \\
6^{\mathrm{h}} 50^{\mathrm{m}} \cdot 3\end{array}$}} & \multirow{2}{*}{$\begin{array}{c}\text { Planet } \\
97 \text { Klotho }\end{array}$} & \multicolumn{4}{|c|}{ Position 1912.0} & \multirow{2}{*}{$\begin{array}{c}\text { Gr. } \\
1 I^{\mathrm{m}} \cdot 5\end{array}$} & I9I 2 & M.Z.Gr. & Planet & \multicolumn{4}{|c|}{ Position 1912.0} & Gr. \\
\hline Mai 3 & & & & I $3^{h}$ & $37^{\mathrm{m}} \cdot \mathrm{o}$ & $+3^{\circ}$ & $22^{\prime}$ & & Mai 8 & $9^{\mathrm{h}} \quad 7^{\mathrm{m}} \cdot 7$ & 424 Gratia & I $5^{\mathrm{h}}$ & $\mathrm{I}^{\mathrm{m}} \cdot 25$ & & $5 I^{\prime}$ & $\mathrm{I} 3^{\mathrm{m}} \cdot 5$ \\
\hline$»$ & & $»$ & 365 Corduba & 13 & 0.5 & -0 & $4 \mathrm{I}$ & I $3 \cdot 5$ & 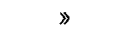 & $»$ & 513 Centesima & 15 & 16.9 & -8 & I I & I 3.8 \\
\hline$»$ & & $\gg$ & 535 Montague & I 3 & 3.6 & +3 & 5 & 12.0 & $»$ & $»$ & I 9 I 2 PC (neu) & 5 & 2.7 & -4 & 3 & 3.5 \\
\hline 》 & & $》$ & $669[1908 \mathrm{DQ}]$ & I 3 & I 2.3 & +2 & 25 & I 4.0 & Juni & $37 \cdot 1$ & 547 Praxedis & 16 & I 1.6 & $-\mathrm{I}$ & 33 & I 3.5 \\
\hline ai 7 & 7 & I $4 . \mathrm{I}$ & $344 \mathrm{De}$ & I 3 & $5^{2 \cdot 3}$ & -3 & I 3 & 10.5 & Juni & 749.2 & 2 I $9 \mathrm{Th}$ & I 7 & 2.6 & -6 & 25 & I I. 5 \\
\hline$»$ & & $\gg$ & $48 \mathrm{I} \mathrm{Em}$ & I 3 & 59.85 & -4 & 2 & I 2.5 & 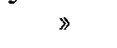 & $\gg$ & 3 I $\mathrm{Ch}$ & 16 & $57 \cdot 7$ & -3 & $5^{6}$ & $1 \mathrm{I} .5$ \\
\hline$»$ & & $»$ & I9I 2 PA (neu) & I 3 & 58.7 & -0 & 22 & 14.0 & $》$ & $\gg$ & 402 Chloë & 7 & 4.6 & -7 & I 7 & I 2.0 \\
\hline$》$ & 9 & I 6.1 & 232 Rus & I 5 & 6.5 & -6 & 7 & 12.5 & $\gtrsim$ & $\searrow$ & 489 Con & I 6 & 42.0 & -3 & I I & I 2.5 \\
\hline$\gg$ & & $»$ & 424 Grat & I 5 & 2.I & -6 & 53 & 3.5 & Juni io & $9 \times 8.8$ & $3 \mathrm{Ju}$ & 7 & I O. I & -4 & I 3 & 10.0 \\
\hline$»$ & & $»$ & $592[190$ & I 4 & 5 I.7 & -4 & I 5 & I 4.0 & $\gg$ & $\gg$ & $3{ }_{3} \mathrm{Ch}$ & 6 & 53 & -3 & $5^{\circ}$ & I I. 5 \\
\hline$»$ & & $»$ & $635[1907 \mathrm{ZS}]$ & I 4 & 44.65 & -5 & 45 & I $3 \cdot 5$ & Juni I 3 & $7 \quad 49.6$ & 329 Sve & 6 & 59.1 & +3 & 48 & I 2.5 \\
\hline$》$ & & $》$ & 19 I 2 PB (neu) & I 5 & 3.5 & -4 & 6 & I 3.5 & $\gg$ & 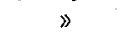 & 49 I Carina & 16 & 41.9 & +3 & 47 & I $3 \cdot 5$ \\
\hline Iai 8 & 9 & $7 \cdot 7$ & 232 Russia & I 5 & 5.65 & -6 & 2 & I 2.5 & & eîs, & 2 Juni & & S & Beljat & sky & \\
\hline
\end{tabular}
Dagegen erhält man mit Hilfe der Gleichung ( 1 ), wenn für $\delta_{m}$ der genaue Wert $+86^{\circ} 25^{\prime} 22^{\prime \prime} 3$ substituiert wird, $p_{m}=$ $82^{\circ} 22^{\prime} 50^{\prime \prime} 2$; der durch die Benutzung der Formel (I) verursachte Fehler beträgt also nahezu 2 Minuten.

Wien, v. Kuffnersche Sternwarte, I 9 I 2 Mai I.

\title{
Photographische Aufnahmen von kleinen Planeten in Simeïs.
}

\section{Bedeckung von $\alpha$ Scorpii durch den Mond 1912 Mai 30.}

Beobachtung auf der Bonner Sternwarte.

Der Eintritt konnte wegen tiefen Standes des Mondes in starkem Dunst nicht beobachtet werden.

Austritt: $\mathrm{I} 5^{\mathrm{h}} \mathrm{I}^{\mathrm{m}} 57^{\mathrm{s}} \mathrm{I}$ Sternzeit Bonn, Beobachter Giebeler am 6 zöll. Heliometer ${ }^{1} 5^{\mathrm{h}} \mathrm{IO}^{\mathrm{m}} 55^{\mathrm{s}} \mathrm{O} \quad 》 \quad 》$. Beob. Mönnichmeyer am 6 zöll. Refraktor von Schröder. Austritt war scharf aufzufassen, Comes nicht zu sehen.
Beobachtung auf der Königstuhl-Sternwarte.

I) Beob. E. Ernst, Kressmannrefraktor $318 \mathrm{~mm}$. Eintritt: $\quad 9^{\mathrm{h}} 30^{\mathrm{m}} 17^{\mathrm{s}} \cdot 79$ M. Z. Kgst. Austritt: Io $47 \quad 4.73$ » 3 chronogr. registriert Eintritt gut, Austritt um $0.4-0.5 \mathrm{zu}$ spät.

2) Beob. F. Kaiser, Bruce-Teleskop, Leitrohr $254 \mathrm{~mm}$. Eintritt: $\quad 9^{\mathrm{h}} 30^{\mathrm{m}} \mathrm{I} 7^{\mathrm{s}} \cdot 4^{2}$ M. Z. Kgst.

Austritt: $1047 \quad 1.64$

Ein- und Austritt gut.

Heidelberg, I 9 I 2 Juni I. A uge- u. Ohrmethode

Bonn, I 9 I 2 Mai $3 \mathbf{I}$.

Inhalt zu Nr. 4589. A. Bemporad. Osservazioni fotometriche di Mira Ceti eseguite nel R. Osservatorio astrofisico di Catania. 69. - A. A. Nijland. Das Mira-Minimum von Januar 1912. 77. - H. Shapley. On the Periods and Secondary Minima of some Variable Stars. 79. - F.W. Dyson. The Presence of Radium in the Chromosphere. 81. - L. de Ball. Berechnung des Positionswinkels eines Bogens, bezogen auf den Mittelpunkt. 83. - S. Beljawsky. Photographische Aufnahmen von kleinen Planeten in Simeïs. 83. - Bedeckung von $\propto$ Scorpii durch den Mond 1912 Mai 30. 83 . 